\title{
Robotic Arm Assisted Total Knee Arthroplasty: Addressing 1-12 Degrees Valgus Knees
}

\author{
Jingwei Zhang ${ }^{1}$, Manoshi Bhowmik-Stoker ${ }^{1}$, Laura Y. Scholl ${ }^{1}$, Caitlin \\ Condrey $^{2}$, Kevin Marchand ${ }^{2}$, Robert Marchand ${ }^{2}$ \\ ${ }^{1}$ Stryker, Mahwah, NJ, USA \\ ${ }^{2}$ South County Hospital, Wakefield, RI, USA \\ jingwei.zhang1@stryker.com, manoshi.bhowmik-stoker@stryker.com \\ laura.scholl@stryker.com, ccondrey@scortho.com, \\ kevin.marchand@me.com, marchand33@cox.net
}

\begin{abstract}
The purpose of this work was to determine the number of soft tissue releases and component orientation of valgus cases performed with Robotic-Arm Assisted total knee arthroplasty (RATKA).

This study was a retrospective chart review of cases performed by a single surgeon from July 2016 to December 2017. 72 RATKA cases were defined as having a valgus deformity preoperatively. Patient demographics and intraoperative surgical details were collected, including initial and final 3D component alignment, knee balancing gaps, full or partial releases. Postoperatively, radiographs, adverse events, and reduced WOMAC pain and KOOS Jr scores were collected at 6 months post-operatively.

Pre-operatively, knee deformities ranged from $1^{\circ}$ to $12^{\circ}$ with fixed flexion contracture. All knees were corrected within 2.5 degrees of mechanical neutral. Medial and lateral gaps were balanced in extension $100 \%$ of cases and flexion $93 \%$ of cases.

Radiographic evidence suggested well seated and well fixed components. No revision and reoperation is reported. Patient reported outcomes measures collected at 6-month follow up indicated an improvement in WOMAC pain score from 9.6 to 3.2 and improvement in KOOS $\mathrm{Jr}$ from 44.7 to 74.4 points.

In this retrospective case review, soft tissue releases were not needed to address valgus knees ranging from $1-12^{\circ}$ of deformity. The surgeon was able to balance the knee with bone resections and avoid disturbing the soft tissue envelope. While this study has a number of limitations, RATKA for valgus knees should continue to be investigated in a multicenter study.
\end{abstract}




\section{Introduction}

Valgus deformity in an end stage osteoarthritic knee can be difficult to correct with no clear consensus on case management. It has been reported that the surgeon must have a detailed understanding of the location, orientation and physical characteristics of the soft tissues providing medial and lateral stability to address the coronal and joint line restoration. Several methods of selective ligamentous releases have been described in literature [1]. Dependent on if the joint can be reduced and the degree of medial laxity or distension, a surgeon must use their discretion on the correct method of lateral releases. It has been shown that the failure to adequately release may leave the patient unable to achieve full extension [2], while over releasing results in joint instability and patient dissatisfaction [3]. To reduce inadvertent soft tissue damage in a valgus case, it has been suggested that precise bone resections could be applied while maintaining the integrity of the soft tissue envelope. Robotic assisted (RA) technology has been shown to have three dimensional (3D) cut accuracy which could assist with addressing these complex cases [4]. The purpose of this work was to determine the number of soft tissue releases and component orientation of valgus cases performed with RA total knee arthroplasty (TKA).

\section{Materials and Methods}

This study was a retrospective chart review of cases performed by a single surgeon from July 2016 to December 2017. The surgeon performed 565 RA TKA of which 72 were defined as having a valgus deformity pre-operatively. Patient demographics and intraoperative surgical details were collected. Initial and final 3D component alignment, knee balancing gaps, and component size were collected intraoperatively (Figure 1). Full or partial releases were documented intraoperatively. For valgus knees, attention was focused on the lateral soft tissues stabilizers including the lateral collateral ligament, popliteal tendon, facia lata, postero-external articular capsule, biceps and external gastrocnemius muscles. Post-operatively, radiographs, adverse events, and reduced WOMAC pain and KOOS Jr scores were collected at 6 months post-operatively per standard of care.

\section{Results}

Pre-operatively, knee deformities ranged from reducible knees with less than $5 \mathrm{~mm}$ of medial laxity to up to $12^{\circ}$ with fixed flexion contracture. All knees were corrected within 2.5 degrees (Figure 2) of mechanical neutral. All cases were adjusted intraoperatively prior to bony resection. In the final plan, average component position of the femur was $0.26^{\circ}$ valgus, $4.07^{\circ}$ flexion, $2.20^{\circ}$ internal rotation, where $68 \%$ of the femur were placed in internal rotation. Average component position of the tibia was $0.37^{\circ}$ valgus, $2.96^{\circ}$ slope, and $0.01^{\circ}$ internal rotation, where all tibial components were either placed with a neutral or valgus orientation. Flexion and extension gaps during knee balancing were within $2 \mathrm{~mm}$ (mean 1 $1 \mathrm{~mm}$ ) for all knees. Medial and lateral gaps were balanced in extension $100 \%$ of cases and flexion $93 \%$ of cases. The average flexion gap was $18.3 \mathrm{~mm}$ and the average extension gap was $18.7 \mathrm{~mm}$. For component size prediction, the surgeon achieved their planned within one size on the femur $93.8 \%$ and tibia $100 \%$ of the time. The surgeon upsized the femur in $6.2 \%$ of cases. Soft tissue releases were reported in one of the cases.

At latest follow-up, radiographic evidence suggested well seated and well fixed components. Radiographs also indicated the patella components were tracking well within the trochlear groove. No revision and re-operation is reported. Patient reported outcomes measures collected at 6-month follow up indicated an improvement in WOMAC pain score from 9.6 to 3.2 and improvement in KOOS Jr from 44.7 to 74.4 points. 


\section{Discussion and Conclusion}

In this retrospective case review, soft tissue releases were not needed to address valgus knees ranging from $1-12^{\circ}$ of deformity. The surgeon was able to balance the knee with bone resections and avoid disturbing the soft tissue envelope. To achieve this balance, it was noticed for many of the valgus knees, the femoral component was oriented with internal and valgus rotations while the tibial component was oriented with valgus rotations. This allowed the surgeon to close the lateral gap and balance it with the medial gap. It was also noticed that the surgeon maintained approximately $18 \mathrm{~mm}$ flexion and extension gaps. Larger gaps can be less desirable for valgus knees since their soft tissue is known to be laxer than that of medial or neutral knees.

As valgus knees can require a greater amount of planning and precision, RA technology may be an option for surgeons to plan the case and make intraoperative component adjustments prior to bone cuts [5]. In literature, RA technology has been shown to reduce in post-operative pain and opioid usage which provides benefits to the patient and the hospital system [6]. This study required no intentional soft tissue releases, indicating RA TKA may reduce post-operative pain. While this study has a number of limitations, RA TKA for valgus knees should continue to be investigated in a multicenter study.

\section{Figures}

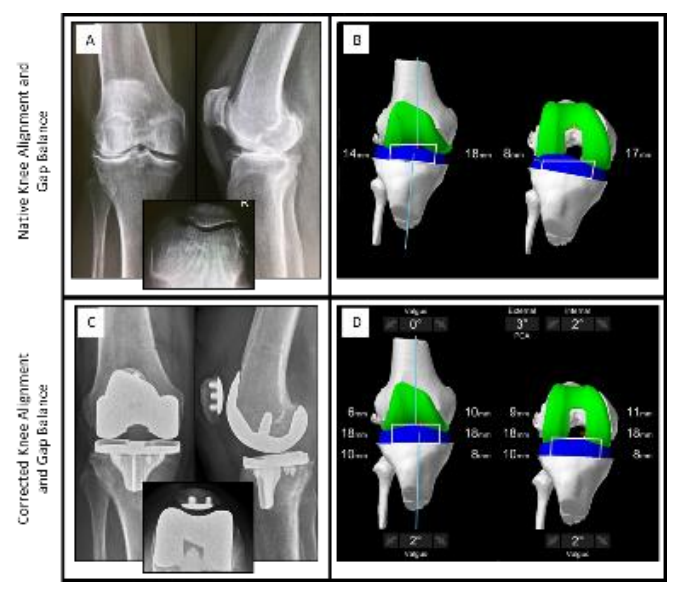

Figure 1. A representative patient with a native valgus aligned knee shown in a preoperative radiograph (a) with unbalance medial and lateral gaps (b). Intraoperative adjustments were made to correct alignment and provide the patient with a neutrally aligned knee (c) with balanced medial and lateral gaps (d). 


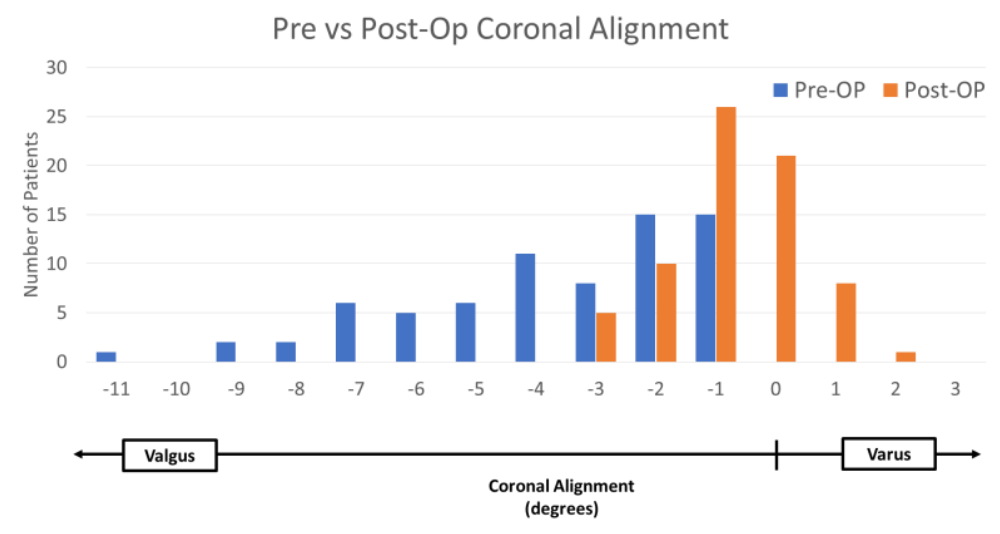

Figure 2: Coronal alignment of cases pre- and post-operative. All patients were restored to be within neutral mechanical alignment.

\section{References}

[1] Whiteside LA., Selective ligament release in total knee arthroplasty of the knee in valgus, Clin Orthop Relat Res. 1999 Oct;(367):130-40.

[2] Meloni MC, Hoedemaeker RW, et. al., Soft tissue balancing in total knee arthroplasty., Joints. 2014 Jan-Mar; 2(1): 37-40.

[3] E. Carlos Rodriguez-Merchan., Instability Following Total Knee Arthroplasty, HSS J. 2011 Oct; 7(3): 273-278.

[4] Hampp EL, Chughtai M, Scholl LY, Sodhi N, Bhowmik-Stoker M, Jacofsky DJ, Mont MA., Robotic-Arm Assisted Total Knee Arthroplasty Demonstrated Greater Accuracy and Precision to Plan Compared with Manual Techniques., J Knee Surg. 2018 May 1.

[5] Marchand RC, Sodhi N, Bhowmik-Stoker M, Scholl L, Condrey C, Khlopas A, Sultan AA, Newman JM, Mont MA., Does the Robotic Arm and Preoperative CT Planning Help with 3D Intraoperative Total Knee Arthroplasty Planning?, J Knee Surg. 2018 Aug 15.

[6] Kayani B, Konan S, Tahmassebi J, Pietrzak JRT, Haddad FS., Robotic-arm assisted total knee arthroplasty is associated with improved early functional recovery and reduced time to hospital discharge compared with conventional jig-based total knee arthroplasty., Bone Joint J. 2018 Jul;100B(7):930-937. 